\title{
Anterior Compartment Syndrome after an Achilles Tendon Repair: A Case Report
}

\author{
Kobayashi Moto', Miyamoto Seiya ${ }^{2}$, Kashiwagura Takeshi ${ }^{3}$, Nozaka Koji ${ }^{4}$, Chida Shuichi ${ }^{1}$, \\ Miyakoshi Naohisa ${ }^{4}$, Shimada Yoichi ${ }^{4}$ \\ ${ }^{1}$ Department of Orthopedic Surgery, Hiraka General Hospital, Yokote, Japan \\ ${ }^{2}$ Department of Orthopedic Surgery, Nakadori General Hospital, Akita, Japan \\ ${ }^{3}$ Department of Rehabilitation, Akita City Hospital, Akita, Japan \\ ${ }^{4}$ Department of Orthopedic Surgery, Akita University, Akita, Japan \\ Email: moto_koba@hotmail.co.jp
}

Received 6 February 2016; accepted 11 March 2016; published 14 March 2016

Copyright (C) 2016 by authors and Scientific Research Publishing Inc.

This work is licensed under the Creative Commons Attribution International License (CC BY). http://creativecommons.org/licenses/by/4.0/

\section{(c) (i) Open Access}

\begin{abstract}
Compartment syndrome may cause irreversible dysfunction if not treated correctly. The occurrence of compartment syndrome is not recognized as a potential complication after Achilles tendon rupture, and only a small number of such cases have been reported. We report the case of a 16-year-old girl with rupture of the right Achilles tendon. On postoperative day 4, she experienced severe anterior ankle pain. A blood test revealed a creatine kinase level of $7976 \mathrm{IU} / \mathrm{L}$; the pressure in the distal anterior compartment was $90 \mathrm{mmHg}$ and proximal compartment was $40 \mathrm{mmHg}$ (needle manometer method). Magnetic resonance imaging (MRI) revealed a signal change in the anterior compartment. Anterior compartment syndrome was diagnosed on the basis of clinical findings, pressure measurements, and MRI findings. Emergency surgery was performed, and the anterior fascia was released. However, the dysfunction in the form of limited range of ankle motion remained. The cause of the compartment syndrome after Achilles tendon rupture was unclear. The patient's involvement in basketball and positioning of the ankle in plantar flexion with a cast might have been contributing factors in our case. We need to consider the possibility that compartment syndrome may occur after Achilles tendon rupture.
\end{abstract}

\section{Keywords}

Achilles Tendon Rupture, Compartment Syndrome, Complication

\section{Introduction}

Compartment syndrome is defined as the situation that the compartmental pressure in a fascial compartment ex-

How to cite this paper: Moto, K., Seiya, M., Takeshi, K., Koji, N., Shuichi, C., Naohisa, M. and Yoichi, S. (2016) Anterior Compartment Syndrome after an Achilles Tendon Repair: A Case Report. Open Journal of Orthopedics, 6, 47-51.

http://dx.doi.org/10.4236/ojo.2016.63007 
ceeds the capillary pressure. This leads to hypoperfusion of intracompartmental tissues and the clinical findings usually show severe pain and positive passive stretch test. If untreated or delayed treatment, it often causes irreversible dysfunction by ischemia. Compartment syndrome complicating a ruptured Achilles tendon has not previously been recognized. We report a case of compartment syndrome occurring after Achilles tendon repair.

\section{Case}

The patient was a 16-year-old girl belonging to a high-school basketball team. She had sensed fatigue of the right lower extremity for several days before falling and experiencing pain in the right ankle region during a basketball practice session. She was unable to perform plantar flexion in her right ankle and was unable to walk independently. She was brought to our hospital by her family.

Her height was $162 \mathrm{~cm}$, body weight was $50 \mathrm{~kg}$, and body mass index was 21.6. Her past medical or surgical history was not remarkable.

At the emergency department, clinical findings showed a positive Thompson test and a palpable defect of the Achilles tendon. Motor and sensory testing of her right leg was normal. No abnormalities existed in her hip and knee.

The diagnosis of a complete tear of the right Achilles tendon was made on the basis of the clinical findings. Surgical and nonsurgical treatment options were offered to her and her family, and they chose the surgical treatment. We informed general methods and complications. They consented to an open repair of Achilles tendon rupture. The leg was immobilized in ankle maximum plantar flexion with a plaster cast.

Achilles tendon repair was performed on the day after the injury under general anesthesia with continuous epidural anesthesia. A pneumatic tourniquet was placed on the right thigh and was inflated to $300 \mathrm{mmHg}$. The patient was placed in the prone position, and a $6 \mathrm{~cm}$ incision was made over the medial edge of the Achilles tendon, centered over the palpable defect of the Achilles tendon. The skin and subcutaneous tissue were dissected sharply down to the level of the paratenon. The operative findings revealed a typical rupture pattern. That is, the Achilles tendon had ruptured completely and both stump ends were ragged with a clot in the gap. A number 2 braided nonabsorbable suture material (Tefdesser; Kono seisakusho, Chiba, Japan) was used to repair the tendon. Three sutures were placed in the proximal section of the tendon using the 6-strand modified Kessler technique ( 1 suture in the dorsal and 2 sutures in the ventral side). Three sutures were placed in the distal segment in a similar fashion. Additional absorbable sutures (2 - 0 Vicryl; Johnson Johnson, Tokyo, Japan) were inserted using a hemi-circumferential cross-stitch method.

The operation was performed by one senior orthopedic surgeon. The surgery time was 82 minutes, and the duration of application of the pneumatic tourniquet was 81 minutes. The leg was immobilized in ankle 30 degree of plantar flexion with a plaster cast.

Postoperative treatment was started according to the usual protocol, and the patient was encouraged to move the toes immediately after the operation. The early postoperative course was uneventful. The patient received 2 doses of antibiotics (CEZ $1 \mathrm{~g}$ ) at 8 - 10 hour intervals postoperatively. She also took a pain killer (oral NSAID) from the day after the surgery.

On the second day after the operation, the patient complained of moderate anterior ankle pain. Examination of the leg revealed no remarkable findings, and the plaster cast was replaced in ankle 10 degree plantar flexion.

On postoperative day 4, the patient complained of severe ankle pain, and the cast was removed. The lower leg was tense, and showed marked erythema of the anterior ankle. The patient could not perform dorsal flexion of the ankle because of the pain. Severe pain also occurred with plantar flexion of the toes and ankle (i.e., the passive stretch test was positive). A blood test showed a creatine kinase level of $7976 \mathrm{IU} / \mathrm{L}$; the pressure in the distal anterior compartment was $90 \mathrm{mmHg}$ and proximal compartment was $40 \mathrm{mmHg}$ (needle manometer method). Magnetic resonance imaging (MRI) revealed a signal change in the anterior compartment (Figure 1). A diagnosis of anterior compartment syndrome was made on the basis of the clinical findings, pressure measurements, and MRI findings. We informed the patient and her family of the unexpected complication that had to be treated immediately, which must be very rare case. She and her family agreed to receive the surgery.

Emergency surgery was deemed necessary, and the patient was taken to the operating room immediately on the same day. General anesthesia was administered, and the operation was performed without use of a pneumatic tourniquet. Two incisions were made over the anterolateral aspect (midway between the anterior edges of the tibia and fibula) of the right lower leg (Figure 2). The proximal incision was approximately $6 \mathrm{~cm}$ in length, and 


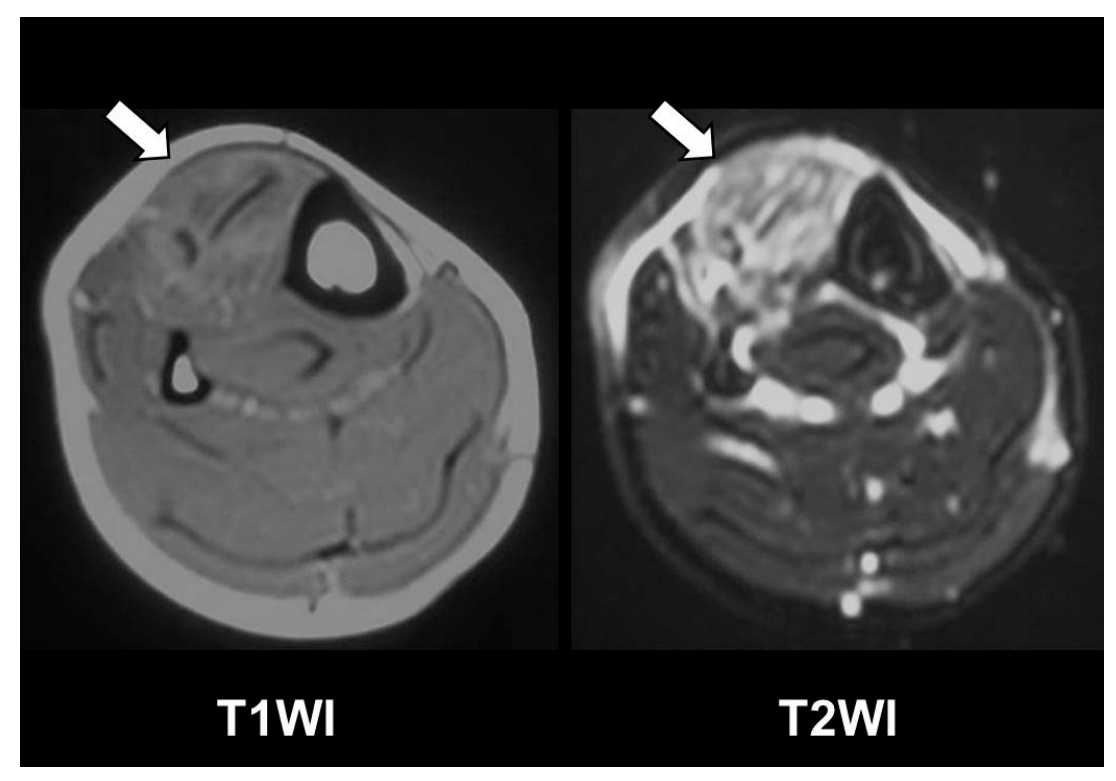

Figure 1. Magnetic resonance image of the right lower leg (postoperative 4 days). White arrow indicates the anterior compartment. Signal change (T1WI; low-iso, T2WI; high) is present.

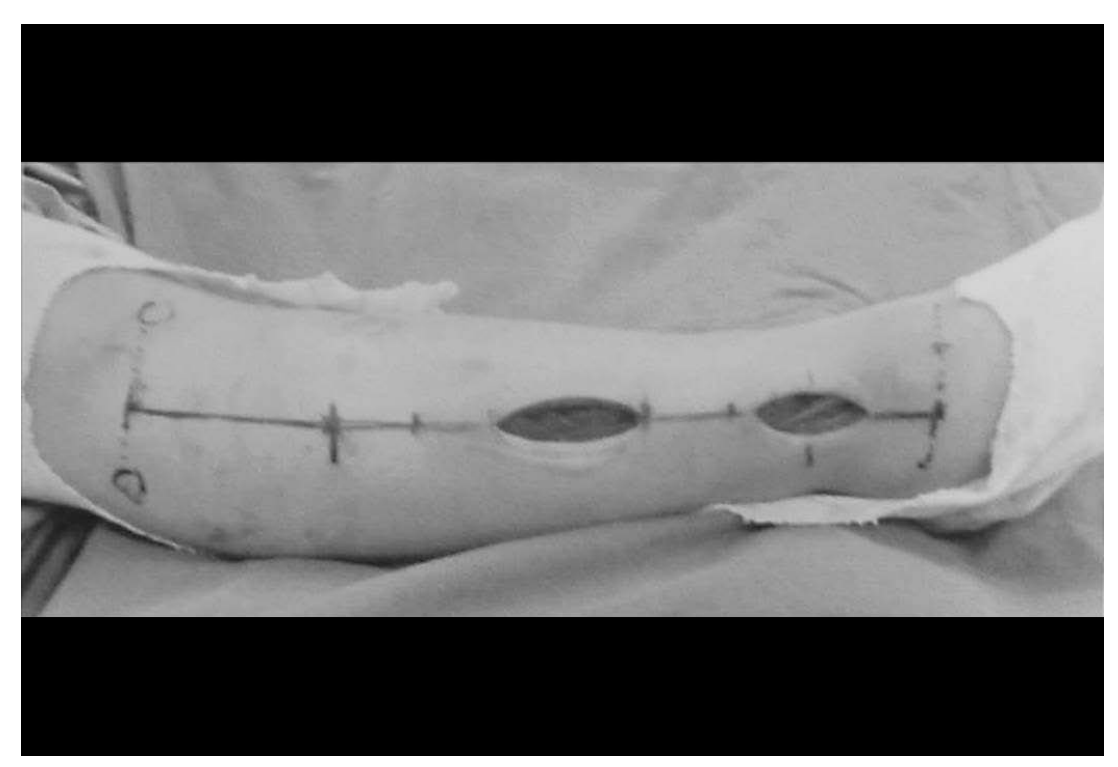

Figure 2. Incisions for the fascia release (lateral aspect of the right lower leg).

the distal approximately $5 \mathrm{~cm}$. The fascia of the anterior compartment was released with 2 incisions, approximately $30 \mathrm{~cm}$ from ankle level to proximal tibia level. The color of the muscle subsequently improved from dark brown to red. The surgical wounds were left as open wounds with wet dressing. After surgery, the leg was immobilized with a splint at moderate ankle plantar flexion.

Muscle strength of the extensor hallucis longus (EHL) and tibialis anterior (TA) improved gradually. An operation to perform wound closure was undertaken 17 days after the surgery. Then the patient was allowed to weight bear with a short leg brace.

Six months after the second surgery, the patient could perform activities of daily living with no problems. She also started playing basketball again, but the shortening of the EHL and TA muscles remained. The passive range of motion of the ankle was 0 degrees of dorsiflexion and 30 degrees of plantar flexion (Figure 3 ). When the patient positioned the ankle in plantar flexion, the toes were passively dorsiflexed. 


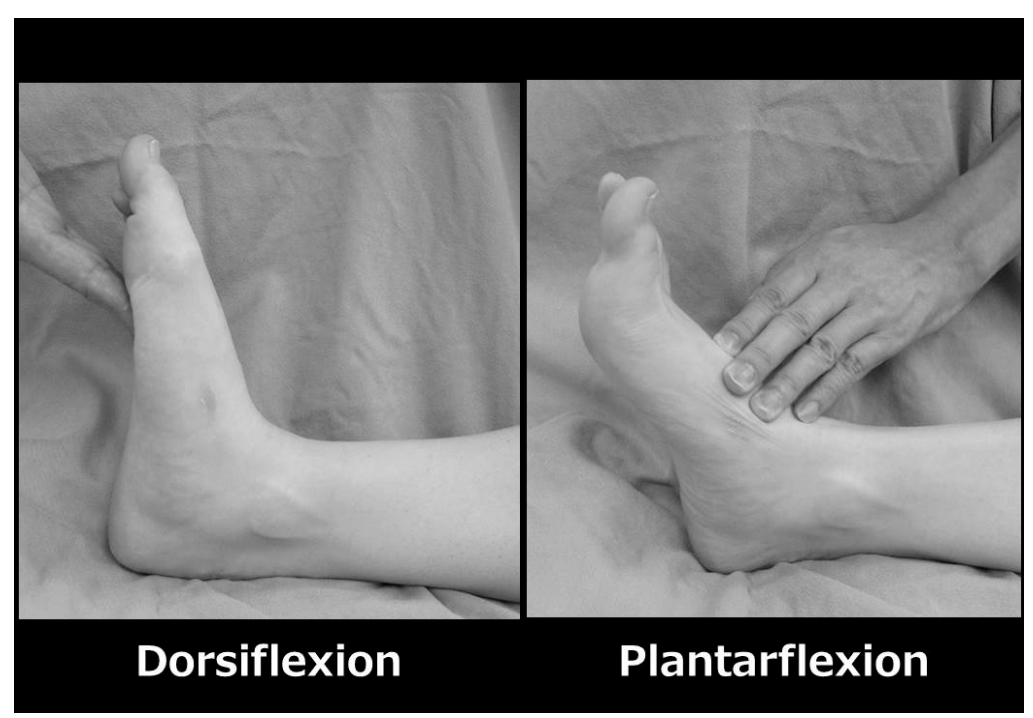

Figure 3. Pictures of the right lower extremity (6 months after fascia release). Passive dorsiflexion (left), passive plantarflexion (right).

\section{Discussion}

Compartment syndrome after rupture of the Achilles tendon has not been described in textbooks, and there are only 3 previously reported cases (Table 1) [1]-[3]. All of these cases were the anterior compartment syndrome. One case occurred during conservative therapy 20 days after injury; 2 cases occurred after Achilles tendon repair, 3 days and 12 days after the repair. The cause of the anterior compartment syndrome was not apparent in any of the 3 cases. The factors considered were patient positioning, use of a pneumatic tourniquet, vasospasm, and anatomical abnormality. These possibilities have not been clarified because of very few cases.

Van Niekerk [3] pointed out the possibility that immobilization of the ankle in plantar flexion with a cast might be a factor in these cases. In addition, Weiner and colleagues [4] reported that the intramuscular pressures in the anterior compartment elevated when the ankle was placed in plantar flexion with a cast, and that the pressures increased 5-fold compared with those without a cast. They concluded that application of a plaster cast after an injury can increase the risk of compartment syndrome. In our case, the ankle had been immobilized in 30 degree plantar flexion place with a cast for about 6 days. This might have been one of the causes. In previous reports, only one case was immobilized by the equinus cast [3]. The other cases of immobilized ankle position were neutral [2] and unknown [1].

On the other hand, Achilles tendon rupture is a common injury in orthopedic surgery, and in spite of the fact that many of patients have been treated by cast immobilization, very few compartment syndrome complications have been reported. We considered that various factors might have played a role in the occurrence of anterior compartment syndrome in our patient. First, she was a basketball player who had muscular leg, and second, she had sensed fatigue of the right lower extremity before rupture of the Achilles tendon occurred. Asymptomatic chronic compartment syndrome might have been present. Kostopoulos et al. [5] in their investigation of male recreational basketball players reported that basketball-simulated activities may significantly increase anterior compartment pressure. The previous 2 cases might have muscular leg; flight instructor in the military [2] and heavy athletic build [3].

When the ankle is immobilized in plantar flexion with a cast in the treatment of Achilles tendon rupture, regardless of whether a conservative or surgical method is employed, we should consider that intra-compartment pressures may increase. Compartment syndrome may cause severe and permanent dysfunction, and we need to inform patients of the possibility of compartment syndrome after Achilles tendon rupture.

\section{Conclusions}

1) We encountered a rare case in which anterior compartment syndrome of the lower leg occurred after surgery for Achilles tendon rupture. 
Table 1. Previous reports.

\begin{tabular}{ccccccc}
\hline Author (Year) & Age/Sex & $\begin{array}{c}\text { Treatment for } \\
\text { Achilles tendon } \\
\text { rupture }\end{array}$ & $\begin{array}{c}\text { Compartment } \\
\text { site and pressure } \\
(\mathrm{mmHg})\end{array}$ & $\begin{array}{c}\text { Diagnosis of } \\
\text { compartment } \\
\text { syndrome }\end{array}$ & $\begin{array}{c}\text { Follow-up } \\
\text { period }\end{array}$ & $\begin{array}{c}\text { Complication by } \\
\text { compartment } \\
\text { syndrome }\end{array}$ \\
\hline $\begin{array}{c}\text { Rankin EA } \\
(1981)\end{array}$ & 29/Female & Operation & $\begin{array}{c}\text { Anterior } \\
(-)\end{array}$ & 12 days & 1 year & Asymptomatic \\
$\begin{array}{l}\text { Reed } \mathrm{J} \\
(2004)\end{array}$ & 41/Male & Operation & $\begin{array}{c}\text { Anterior } \\
80.7\end{array}$ & 3 days & 6 months & Drop toe \\
$\begin{array}{c}\text { Van Nielerk M } \\
(2011)\end{array}$ & 45/Male & $\begin{array}{c}\text { Conservative } \\
\text { therapy }\end{array}$ & $\begin{array}{c}\text { Anterior } \\
110\end{array}$ & 20 days & 6 weeks & Droot \\
$\begin{array}{c}\text { Kobayashi } \\
(2016)\end{array}$ & 16/Female & Operation & $\begin{array}{c}\text { Anterior } \\
90\end{array}$ & 4 days & 1 year & Ankle contracture \\
\hline
\end{tabular}

*Days after surgery or injury of Achilles tendon rupture.

2) Although fascia release was performed for the anterior compartment syndrome at an early stage, the dysfunction remained.

\section{References}

[1] Rankin, E.A. and Andrews G. (1981) Anterior Tibial Compartmental Syndrome: An Unusual Presentation. Journal of the National Medical Association, 73, 1156-1157.

[2] Reed, J. and Hiemstra, L.A. (2004) Anterior Compartment Syndrome Following an Achilles Tendon Repair: An Unusual Complication. Clinical Journal of Sport Medicine, 14, 237-241. http://dx.doi.org/10.1097/00042752-200407000-00007

[3] Van Niekerk, M., Jawad, F., Huntley J.S. and McCowan, S. (2011) A 45-Year-Old Man Presenting with Anterior Compartment Syndrome Three Weeks Following Conservative Treatment of an Achilles Tendon Rupture. Scottish Medical Journal, 56, 1-3. http://dx.doi.org/10.1258/smj.2011.011103

[4] Weiner, G., Styf, J., Nakhostine, M. and Gersuni D.H. (1994) Effect of Ankle Position and a Plaster Cast on Intramuscular Pressure in the Human Leg. The Journal of Bone \& Joint Surgery, 76, 1476-1481.

[5] Kostopoulous, N., Fatouros, I.G., Siatitsas, I., Baltopoulos, P., Kambas, A., Jamurtas, A.Z. and Fotinakis, P. (2004) Intense Basketball-Simulated Exercise Induces Muscle Damage in Men with Elevated Anterior Compartment Pressure. Journal of Strength Conditioning Research, 18, 451-458. 